\title{
UNA PROPUESTA REYISTA PARA LA JUVENTUD DEL PAÍS: \\ la Segunda Reserva del Ejército Nacional EN EL Distrito Federal, 1900-1902
}

\author{
Marco Enrique Sánchez López*
}

\section{Resumen}

Este artículo se acerca al proyecto más emblemático de los impulsados por el general Bernardo Reyes mientras tuvo a su cargo la Secretaría de Guerra y Marina del gobierno mexicano (1900-1902): la creación de la Segunda Reserva del Ejército Nacional. Hace un seguimiento de la convocatoria y trabajos de promoción para el alistamiento de jóvenes reservistas $y$, sobre todo, de la importante respuesta que dicha convocatoria tuvo en la capital del país. Asimismo, identifica la presencia de personajes reconocidos en la vida política de la época como promotores de la Segunda Reserva, así como los nombres de oficiales recién enrolados que figurarían en la política nacional años más tarde, lo que da cuenta de la relevancia del proyecto y del impacto que comenzaba a tener cuando fue cancelado.

Palabras clave: Bernardo Reyes, Segunda Reserva del Ejército Nacional, jóvenes reservistas.

\begin{abstract}
This article approaches the most emblematic project those driven by General Bernardo Reyes who was in charge of the Secretary of War and Navy of the Mexican government (1900-1902): the creation of the second national army reserve. It does a follow-up of the summons and works of promotion for the enrollment of young reservists and, especially, of the important response that the above mentioned summons had in the capital of the country. Likewise, he identifies the presence of prominent figures recognized in the political life of the epoch as promoters of the Second Reserve, as well as the officials' names newly enlisted that would appear in the national politics years
\end{abstract}

* Maestría en historia. Instituto Mora 
later, which realizes of the relevancy of the project and of the impact that began to have when it was cancelled.

Keywords: General Bernardo Reyes, second national army reserve, secretary of war and navy.

En enero de 1900, tras la muerte del general Felipe Berriozábal, secretario de Guerra y Marina, el presidente Porfirio Díaz nombró al general Bernardo Reyes como nuevo secretario. Hasta el momento, el general Reyes había sido gobernador del estado de Nuevo León y jefe de la zona militar del noreste de México, donde había mostrado cualidades de liderazgo y destacado como buen administrador público. ${ }^{1}$ Sin embargo, los retos que habría de enfrentar como secretario de Guerra serían mayores. Lo fueron por dos razones. Primero, porque las filas armadas mexicanas estaban lejos de ser un Ejército moderno; el sistema de reclutamiento de la tropa seguía siendo la aborrecida leva; la soldadesca carecía de la mínima instrucción escolar y recibía mala paga; buena parte de la oficialidad tampoco tenía una formación de colegio militar, con el agravante de que las condiciones materiales del ejército eran bastante malas; además, el mando de las fuerzas auxiliares se encontraba diluido entre el gobierno nacional y los de los estados de la república.

La segunda razón por la que el reto de asumir el cargo de secretario de Guerra resultaba tan grande tenía que ver con la sucesión presidencial de 1904. Al iniciar el siglo xx, el presidente Díaz tenía ya 70 años de edad, y los grupos políticos se preocupaban por quién atendería mejor sus intereses en caso de que faltara. En aquellos años, los candidatos principales para suceder a don Porfirio eran, por un lado, el secretario de Hacienda, José Yves Limantour —líder de los "científicos", un grupo político integrado

1 De hecho, más allá de sus tareas en Nuevo León, Reyes se mantuvo siempre muy atento a los problemas de las filas nacionales. De ello hay más de un ejemplo: en 1896, mantuvo comunicación con el entonces secretario Berriozábal en torno a una propuesta de reorganización de la caballería del Ejército nacional; un poco antes, en 1894, momento en que pareció inminente una guerra con Guatemala, Reyes pidió que se le permitiera formar parte de la proyectada fuerza expedicionaria mexicana, y una vez que el presidente Díaz lo autorizó, solicitó todos los mapas y datos sobre la geografía y topografía de la frontera sur del país, demostrando con esto diligencia y compromiso. Niemeyer, El General Bernardo Reyes, p. 92. 
por profesionistas y ligado a los intereses del centro de México-; y por otro, Bernardo Reyes, personaje con gran fuerza política en la poderosa región industrial del norte del país. Al parecer, el presidente Díaz buscó un acuerdo entre ellos, de manera que su sucesión se resolviera como una combinación Reyes-Limantour y se evitara así una confrontación entre ambos. $^{2} \mathrm{Al}$ margen de que ese acuerdo se haya logrado en algún momento, tan cierto es que Reyes y los "científicos" encabezaban fuerzas políticas encontradas que competían por el control del poder nacional, como que las acciones de Reyes, en calidad de secretario de Guerra, los alejarían todavía más. Los conflictos entre grupos con la perspectiva de la sucesión presidencial terminaron por costarle a Bernardo Reyes su cargo al frente de la Secretaría de Guerra. Tuvo que renunciar al puesto en 1902, a escasos dos años de haberlo asumido, y regresar al frente del gobierno de Nuevo León.

Durante los dos años que el general Bernardo Reyes fue secretario de Guerra y Marina, emprendió importantes reformas para atender los problemas de las fuerzas armadas. ${ }^{3}$ Uno de sus proyectos más ambiciosos y emblemáticos fue la creación de la Segunda Reserva del Ejército: un cuerpo armado que dependería directamente del gobierno nacional, proporcionaría una instrucción militar a los civiles voluntarios que se sumaran a él y ofrecería un espacio de participación a grupos de jóvenes de clase media en busca de alternativas para incorporarse a la vida pública del país. Este proyecto fortalecía al Ejército, pero también daba fuerza política a Reyes, por lo que causó recelo entre sus contrincantes y, muy posiblemente, fue el elemento que precipitó su salida de la Secretaría de Guerra. La forma en que se impulsó la creación de este cuerpo reservista en el Distrito Federal y la importante respuesta que obtuvo en la entidad son el objeto de estudio del presente artículo. Estudios de caso como éste permitirán entender mejor la

2 Es posible que este acuerdo se haya logrado en 1900, aunque, si lo hubo, duró poco. Véase Reyes, De mi vida, p. 23.

3 Entre las reformas comenzadas por Reyes estuvo una administrativa, más aumentos salariales, creación de escuelas para la tropa y redefinición de zonas militares. Con medidas como éstas comenzó a enfrentar los retos de modernizar al ejército, aunque su renuncia al cabo de dos años impidió llevarlo a cabo. Sobre este tema véase Sánchez López, "La Segunda Reserva...", 139 pp. 
naturaleza y significado del llamado de Reyes a formar la Segunda Reserva del Ejército Nacional. ${ }^{4}$

\section{El proyecto reyista de la Segunda Reserva}

Con Bernardo Reyes al frente de la Secretaría de Guerra se llevaron a cabo reformas importantes. Una primera medida para reestructurar al Ejército Nacional fue la promulgación de una nueva Ley Orgánica. Reyes había recibido una armada con milicias y guardias nacionales sujetas más a las autoridades estatales que a la propia Secretaría de Guerra. Con esta nueva ley, Reyes intentó someter a todos los cuerpos armados del país al control del gobierno nacional. En este nuevo ordenamiento se hacía mención ya del proyecto más ambicioso del nuevo secretario de Guerra: la creación de la Segunda Reserva.

La Segunda Reserva del Ejército Nacional contaría con las antiguas guardias nacionales organizadas por los estados para sus cuerpos de tropa, con lo que se afianzaba la autoridad de la Secretaría de Guerra sobre este conjunto de fuerzas regionales; de manera paralela, para comandar a las tropas de reserva, se crearía una nueva oficialidad de ciudadanos voluntarios. ${ }^{5}$

La Segunda Reserva fue un cuerpo organizado para ser movilizado sólo en caso de guerra contra una nación extranjera. En tal circunstancia, el gobierno nacional podría poner en pie de guerra, en muy corto tiempo, a grandes contingentes armados. En un eventual conflicto militar con país extranjero, la Segunda Reserva multiplicaría los efectivos del Ejército en breve tiempo. De esta manera, la nueva fuerza reservista permitiría movilizar 76,000 hombres en 45 días, esto es, el triple de los que el Ejército podía disponer hasta antes de la creación de la Segunda Reserva. Este nuevo cuerpo generaría menos gastos para el gobierno nacional de los que requeriría el

\footnotetext{
4 Existen magníficas valoraciones del significado de la Segunda Reserva que nos introducen en el estudio de esta institución, como los trabajos de Alicia Hernández. Sin embargo, estudios de caso como el presente podrán enriquecer esas miradas. En este artículo nos acercamos a la respuesta a la convocatoria para formar la Segunda Reserva específicamente en la capital del país; otros trabajos, como el de Luis Ignacio Sánchez Rojas, se han acercado a la Segunda Reserva en el estado de Veracruz. Hernández Chávez, "Origen y ocaso..."; Hernández Chávez, La tradición republicana...; Sánchez Rojas, "La Segunda Reserva...”.

5 "Ley Orgánica del Ejército Nacional”, arts. 234-236, 240. La Ley puede consultarse en Dublán y Lozano, Colección Legislativa, pp. 755-819.
} 
sostenimiento de un gran ejército permanente $y$, a su vez, instruiría a civiles en los deberes militares para atender una emergencia nacional. ${ }^{6}$ Un mando centralizado y la oportunidad para un sector de ciudadanos de participar en la vida pública a través de un cuerpo militar especial, comprometido con la defensa de la patria frente a amenazas extranjeras, dieron a la Segunda Reserva una gran proyección nacional.

El nuevo cuerpo de oficiales reservistas propuesto por Reyes abría la puerta a actividades públicas a un sector de la sociedad civil, particularmente a jóvenes con cierto nivel de instrucción escolar, que no parecía encontrar sitio en la administración pública ni oportunidades significativas para hacer una carrera política. Los aspirantes debían cumplir con ciertos requisitos de sexo — sólo se aceptaban hombres—, de edad —entre 21 y 45 años—, de buena salud y de cierta escolaridad — debían aprobar un examen de ingreso que demandaba más que la instrucción primaria, así como ser capaces de adquirir conocimientos de alguna complejidad sobre el arte de la guerra—. ${ }^{7}$ Estos ciudadanos voluntarios recibirían instrucción castrense todos los domingos, de manera de que, si fueran llamados a combate, pudieran funcionar como oficiales, fundamentalmente de los cuerpos tácticos del Ejército - infantería y caballería. ${ }^{8}$

6 Además, según sugiere la historiografía, la Segunda Reserva pudo haber sido un primer paso para un proyecto más ambicioso: la instauración del servicio militar obligatorio. El buen recibimiento de la Segunda Reserva podría haber ayudado a llevar a cabo este proyecto, alejando del imaginario colectivo en México la creencia de que el Ejército era sinónimo de pobreza y castigo (creencias asociadas al tradicional y poco popular sistema de reclutamiento por leva). Reyes, De mi vida, p. 27.

7 La mayoría de edad no era un requisito inflexible, pues los menores de veintiún años interesados en ingresar a la Segunda Reserva podrían hacerlo: sólo debían agregar a su solicitud que tenían el consentimiento de sus padres o tutores para ser reservistas. Por lo que toca al tipo de conocimientos y niveles escolares requeridos, la ley era poco explícita. Sin embargo, la Circular núm. 290 de la Secretaría de Guerra, del 5 de febrero de 1901, especificaba las materias que los aspirantes a oficiales reservistas debían dominar para su admisión. Para preparar su ingreso a la reserva, los jóvenes solicitantes debían estudiar materias que los familiarizaran con las ordenanzas del Ejército, obligaciones y órdenes generales; con tácticas militares y prácticas propias de un escuadrón de caballería o de una compañía de infantería; con técnicas de fortificación y asignaturas de topografía y geografía. Reyes, Memoria... 1900-1901, p. 90.

8 En un principio la Segunda Reserva sólo contemplaba la formación de oficiales que servirían a los cuerpos tácticos de infantería y caballería. Sin embargo, avanzado el proyecto, se expandieron las posibilidades de acción hacia los servicios de ingeniería, telégrafo, médico, farmacéutico y veterinario. Reyes, Memoria... 1900-1901, pp. 93-94. 
De esta manera, la Segunda Reserva era una salida a exigencias del Ejército frente a un eventual conflicto militar con el extranjero, ${ }^{9}$ pero era también, y quizás principalmente, una respuesta política a las presiones a favor de espacios para participar en la vida pública. Estas presiones eran ejercidas por una nueva generación de grupos medios, nacida en los años de paz y moldeada por ellos, que más temprano que tarde remplazaría a los generales y políticos de viejo cuño —-muchos de ellos auténticos caciques. ${ }^{10}$ Según Rodolfo Reyes — hijo del general Bernardo Reyes y miembro de esa generación joven-, de haberse desarrollado conforme al proyecto del secretario de Guerra, la Segunda Reserva hubiera podido funcionar como una válvula de escape para la presión que esas nuevas fuerzas representaban para el gobierno. La Segunda Reserva se presentó como una institución que podía dar voz y prestigio a los voluntarios que la formaran. A partir de la instrucción que recibieran, se convertirían en oficiales y suboficiales que se integrarían a los cuerpos tácticos del Ejército y, aunque en menor medida, también a los cuerpos técnicos de artilleros e ingenieros, así como a los de sanidad y telegrafía militar de los Servicios Especiales. En principio, los reservistas no serían tropa común; sin embargo, si bien el proyecto inicial consideraba sólo el ingreso de candidatos con cierto nivel escolar que alcanzarían niveles de oficiales reservistas, parece ser que el entusiasmo despertado atrajo a otros sectores sociales y la convocatoria terminó por abrirse también, con menores exigencias, para formar cuerpos de tropa. El decreto presidencial núm. 257, del 12 de marzo de 1902, permitió que jóvenes de otros sectores sociales, como los alumnos de las Escuelas de Artes y Oficios y los obreros de casas industriales, se integraran a la Segunda Reserva, aunque no como oficiales, sino sólo como sargentos y cabos, respectivamente. ${ }^{11}$ En 1900, cuando el presidente Díaz alcanzaba sus 70 años de edad, el nuevo cuerpo reservista podía haber funcionado como una institución de enlace entre la autoridad política y una parte de la sociedad,

9 La creación de la Segunda Reserva parecía seguir una experiencia similar a la puesta en práctica por el Ejército alemán: un cuerpo de voluntarios llamado Landwehr. En México se conocía esa experiencia alemana. Por ejemplo, desde 1873, había sido publicado un libro que describía todo el sistema militar prusiano. Véase Benavides, La Prusia Militar.

10 Knight, La Revolución Mexicana, p. 49.

11 "El General Díaz, el $1^{\circ}$ de abril de 1902, al inaugurar el $20^{\circ}$ Congreso de la Unión, el segundo periodo del segundo año de sus sesiones", en Los presidentes de México, p. 635. 
en particular la constituida por esa juventud deseosa de participación en la "cosa pública". ${ }^{12}$

El proyecto de la Segunda Reserva parece haber sido recibido con gran entusiasmo por algunos sectores sociales. Tanto así que, según refiere uno de los biógrafos de Bernardo Reyes, el historiador E.V. Niemeyer, los ciudadanos voluntarios que se sumaban a ella convertían sus entrenamientos en eventos sociales y hacían de ellos una auténtica fiesta civil:

Cada domingo los reservistas recibían entrenamiento en los campos locales de maniobras [...]. Además de la instrucción militar, había desfiles, simulacros de guerra, discursos patrióticos y bandas de música. Estos actos se convertirían en un acto social cuando las familias de los participantes asistían trayendo comida que se servía después de las actividades del día. Nunca hubo en tiempo de paz tal patriotismo y devoción al país como los que entonces se observaron. ${ }^{13}$

La convocatoria para crear la nueva oficialidad de la Segunda Reserva parece haber tenido gran eco en todo el país; lo tuvo, ciertamente, en el Distrito Federal.

\section{La promoción de la Segunda Reserva}

La primera acción del gobierno para poner en marcha el proyecto de la Segunda Reserva fue la publicación misma de la Ley Orgánica del Ejército Nacional, en noviembre de $1900 .{ }^{14}$ Esta publicación fue el punto de partida para la creación, unos meses más tarde, de una agrupación promotora del proyecto reservista, con un órgano de prensa propio. Este grupo, sus

12 Reyes, De mi vida, p. 35.

13 Neimeyer, El General Bernardo Reyes, p. 104. La convivencia en la Segunda Reserva forjaba lazos importantes entre sus integrantes. Al respecto decía la Revista México Militar: "Esos grupos [clubes de reservistas] están formados por profesionales de justa reputación como inteligentes y honorables; entre ellos ya existían los lazos del compañerismo escolar o de carrera, y los recuerdos no lejanos, de la época más dichosa de la vida, se despiertan y avivan ante el nuevo compañerismo en una tendencia tan grande y tan noble, como la que les anima, al someterse al aprendizaje de los reclutas." "Oficiales Reservistas", Revista México Militar, vol. 1, Núm. 23, $1^{\circ}$ de mayo de 1901, pp. 496-497.

14 Diario Oficial de la Federación, 11 al 19 de noviembre de 1900. 
comités en los estados y su periódico hicieron una labor importante para convocar a la juventud mexicana a incorporarse a la Segunda Reserva.

De esta manera, en marzo de 1901 se creó el Círculo de Propaganda de la Institución de Oficiales Reservistas. Su objetivo fue dirigir la organización, propaganda y difusión del proyecto reservista a lo largo y ancho del territorio nacional. El Círculo promotor tuvo su sede en el Distrito Federal y estuvo encabezado por un conjunto de civiles, todos ellos personalidades con un reconocimiento ya en la política nacional y, seguramente, algunos de los primeros en haberse enlistado en la Segunda Reserva. El presidente del Círculo era el abogado y profesor de la Escuela Nacional de Jurisprudencia José Mónico Antonio Ramos Pedrueza, personaje muy cercano al general Reyes y al que la historiografía le atribuye la idea original de la creación de la Segunda Reserva. ${ }^{15}$ Como secretario del Círculo fungió el también abogado Joaquín Manuel Villada y Cardoso, hijo del general José Vicente Villada, entonces gobernador del Estado de México; ${ }^{16}$ los cuatro vocales del Círculo fueron Alonso Rodríguez Miramón, Francisco Martínez López, Jesús F. Nieto y el licenciado Heriberto Barrón, tres de los cuales eran o habían sido diputados federales y uno, además, era profesor de la Escuela Nacional Preparatoria. ${ }^{17}$

15 Boletín del Oficial Reservista, año 1, núm. 1, 28 de abril de 1901. Sobre la "idea original” de Ramos Pedrueza para la formulación del proyecto reservista, los biógrafos de Reyes refieren una anécdota, de acuerdo con la cual, en una reunión social, Ramos Pedrueza le propuso a Reyes la creación de una segunda reserva, a la manera de la del Ejército alemán; Reyes pidió un informe detallado sobre la propuesta y, finalmente, parece haber fusionado las ideas de Ramos Pedrueza con las propias, expuestas tiempo atrás en su Ensayo sobre un nuevo sistema de reclutamiento (1885). Así se dio forma al proyecto de la Segunda Reserva del Ejército Nacional. En 1901, Ramos Pedrueza era profesor de la Escuela de Jurisprudencia y diputado federal - tenía ese cargo desde 1898, lo conservaría hasta 1906 y volvería a ser electo diputado federal de 1908 a 1912. González Oropeza, Los diputados de la nación, http:/ / www.diputados.gob.mx/cedia/biblio/virtual/dip/dipna.htm [consultado el 2 de noviembre 2013].

Véanse también Niemeyer, El General Bernardo Reyes, pp. 103-104; Benavides Hinojosa, Bernardo Reyes, pp. 244-245; González de Arellano, Bernardo Reyes, p 45; Reyna Hinojosa, El general Bernardo Reyes, pp. 159-160.

$16 \mathrm{Al}$ momento de comprometerse con el Círculo, Joaquín Manuel Villada era diputado local suplente en el Estado de México — tuvo este cargo de 1900-1902 - y, más adelante, de 1910 a 1912, llegó a ser diputado federal. Legislaturas y Legisladores del Estado de México,

http://148.215.202.57/inesle/PUBLICACIONES\%20INESLE/Libros\%20Publicados/ legislatutas $\% 20 \mathrm{y} \% 20$ legisladores $\% 20 \mathrm{del} \% 20$ edomex.pdf [consultado el 27 de octubre 2013]; González Oropeza, Los diputados de la nación, http://www.diputados.gob.mx/cedia/biblio/ virtual/dip/dipna.htm [consultado el 2 de noviembre 2013].

17 Rodríguez Miramón, diputado federal en 1888-1890 y de nuevo de 1892 a 1906, era el profesor de la Escuela Nacional Preparatoria; Martínez López, diputado federal suplente por 
Barrón, junto con Francisco Martínez López, fueron seleccionados por el propio Círculo, en 1901, para viajar por el territorio nacional y promover la creación de comités en los diferentes estados del país. ${ }^{18}$

Como todo comité promotor de un proyecto abierto, este grupo requirió de un órgano de prensa que funcionara como su vocero. De esta manera, el 28 de abril de 1901 apareció el primer número de El Boletín del Oficial Reservista. Órgano del Círculo de Propaganda de la Institución de Oficiales Reservistas. ${ }^{19}$ Se trataba de una publicación gratuita, de aparición esporádica, dedicada a hacer propaganda en favor de la Segunda Reserva. Transmitía noticias relacionadas con la organización de las diversas agrupaciones de reservistas en el país, así como disposiciones y diferentes circulares de la Secretaría de Guerra dirigidas a la "juventud ilustrada del país", que era el sector de la población al que el Círculo se había propuesto llegar e incorporar al proyecto reservista.

La Ley Orgánica del Ejército se emitió en octubre de 1900, pero se tenía contemplado que entrara en vigor a partir del segundo semestre del siguiente año. ${ }^{20}$ Con todo, la difusión de la convocatoria, la campaña

los estados de Guerrero (1892-1894, 1896-1898), Chihuahua (1894-1896), Estado de México (1896-1898), Michoacán (1896-1898), Sinaloa (1896-1898), y diputado propietario por el Estado de México (1898-1904) y por Michoacán (1904-1906); Heriberto Barrón, diputado suplente por Chihuahua (1908-1910), Hidalgo (1894-1896, 1900-1904), Jalisco (1894-1896) y el Distrito Federal (1904-1906). Barrón sería luego uno de los principales promotores del reyismo frente a la sucesión presidencial de 1910. González Oropeza, Los diputados de la nación, http://www.diputados.gob.mx/cedia/biblio/virtual/dip/dipna.htm [consultado el 7 de noviembre de 2013]; AHUNAM, sección: expedientes de alumnos, serie: Archivo General, exp.1597, 16201. "Los oficiales reservistas. Academias en la Escuela de Tiro de San Lázaro.", El Imparcial, 19 de marzo de 1901, p. 3.

18 En abril de 1901, el primer número de su boletín oficial ya daba noticias de los comités creados en Nuevo León, Guanajuato, Estado de México, Colima y Puebla. En la capital del país, el propio Círculo debe haber funcionado como promotor directo de la Segunda Reserva, porque no se tiene registro de la creación de comités secundarios en la entidad. Boletín del Oficial Reservista, año 1, núm. 1, 28 de abril de 1901.

19 Boletín del Oficial Reservista, año 1, núm. 1, 28 de abril de 1901. Este ejemplar se encuentra en el Archivo General del Estado de Veracruz, Secretaría General de Gobierno - Gobernación y Justicia - Guerra y Marina, Corresp., Xalapa, caja 48. Se trata del único ejemplar que he localizado, ignoro cuántos números llegaron a imprimirse. Igualmente, publicada bajo los auspicios de la Secretaría de Guerra, había una revista de mayor proyección que, entre 1901 y 1902, promovió también el proyecto reservista: La Revista México Militar. Esta publicación quincenal puso al servicio del Círculo de propaganda toda una sección especialmente dirigida a los aspirantes a reservistas.

20 "El General Díaz, el $1^{\circ}$ de abril de 1901, al abrir el $20^{\circ}$ Congreso de la Unión, el segundo periodo del primer año de sus sesiones”, en Los presidentes de México, p. 607. 
para convencer de la importancia del proyecto para el país y los esfuerzos encaminados a persuadir a los jóvenes para alistarse en el Distrito Federal comenzarían desde noviembre de 1900. En esta campaña participó de manera muy destacada el diario oficialista El Imparcial. Por ejemplo, desde finales de noviembre, este periódico hablaba de los beneficios que tenía ingresar a la Segunda Reserva:

Los oficiales reservistas obtienen, una vez aceptados, los honores y consideraciones debidas a su grado y tienen facultad para usar el uniforme correspondiente. De esa manera, además de tener un puesto honroso, en caso de ser llamados al servicio, ingresarán con el grado que les corresponde. ${ }^{21}$ Es indiscutible que el país obtendrá también grandes ventajas, pues para caso necesario, contará con un grupo de oficiales reservistas iniciados en la ciencia militar, y para que de antemano estén hechos en la confraternidad y el compañerismo, tan útiles en el servicio. ${ }^{22}$

Hacia el mes de marzo de 1901, las tareas del Círculo de Propaganda encabezado por Ramos Pedrueza iban encaminadas de buena manera, por lo menos en el Distrito Federal. El Imparcial y la Revista México Militar publicaron los artículos "Los oficiales reservistas" y "La defensa de la patria. Oficiales reservistas", respectivamente, en los que se insistía en la buena respuesta que estaba teniendo la convocatoria en el Distrito Federal y se hacía público un manifiesto suscrito por alumnos de escuelas de estudios superiores como la Escuela Nacional de Jurisprudencia, la Nacional de Ingenieros y la Escuela de Comercio. Este manifiesto, en el que se declaraba apoyo total al proyecto reservista, iba acompañado de un listado de nombres de alumnos de las primeras dos escuelas mencionadas que, en un principio, se registrarían como aspirantes a subtenientes de la Segunda Reserva.

21 Efectivamente, la Ley Orgánica del Ejército Nacional garantizaba una serie de beneficios a quienes ingresaran a las reservas. Aseguraba, desde luego, la expedición de patentes o nombramientos como oficiales por parte de la Secretaría de Guerra; además, otorgaba a los reservistas el derecho a utilizar un uniforme especial. Por otra parte, a los oficiales reservistas también se les abría la oportunidad de pasar al ejército permanente conservando su grado. Reyes, Memoria... 1900-1901, pp. 87-89, 91.

22 "Los oficiales reservistas en la nueva organización del Ejército", El Imparcial, 26 de noviembre de 1900 , p. 3. 
Más allá de la propaganda en la prensa y de la que seguramente se practicó en las escuelas preparatorias y superiores - las listas publicadas y el que profesores de estas escuelas, como Ramos Pedrueza y Alonso Rodríguez, fueran directivos del Círculo de Propaganda, hace pensar que el proyecto reservista se promovía con especial ánimo en esos espacios-, el gobierno hizo su propia labor de promoción de manera directa. Con esta intención, la Secretaría de Guerra emitió circulares dirigidas a los gobernadores para que impulsaran la Segunda Reserva; también publicó manuales que apoyaban el camino de los solicitantes. Este fue el caso, por ejemplo, de "unos pequeños tratados que se ocupan de exponer en términos claros y concisos las materias indispensables para optar el grado de oficial reservista, mediante el examen correspondiente". ${ }^{23}$ El Manual del oficial subalterno, escrito conforme a las últimas disposiciones contenidas en la Ley Orgánica del Ejército para que todo ciudadano interesado en engrosar las filas reservistas pudiera hacerse del conocimiento necesario, incluso de manera autodidacta, fue un buen ejemplo. ${ }^{24}$

La mencionada obra era más que "un pequeño tratado", pues constaba de dos volúmenes — en realidad eran tres, pero el aspirante sólo debía adquirir dos, dependiendo del cuerpo al que estuviera interesado en ingresar-. El primero de los volúmenes ofrecía conocimientos comunes para las armas de infantería y caballería; el segundo y tercero se especializaban en cuestiones referidas a cada una de estas armas en lo particular. Se vendían los manuales y, además, la Revista México Militar los resumía y contribuía de esa manera a su difusión.

Efectivamente, los manuales no eran gratuitos: se vendían. Y no podían ser tan baratos, pues estaban impresos "en un buen papel y encuadernados a la holandesa." ${ }^{25}$ En el Distrito Federal, podían adquirirse en las bibliotecas de la Secretaría de Guerra, por el precio de cincuenta centavos cada tomo. $\mathrm{Al}$ parecer, éste era un precio más bajo que el real, lo que tenía la intención de facilitar su adquisición para la instrucción autodidacta de los jóvenes

23 "Los oficiales reservistas", El Imparcial, 16 de enero de 1901, p. 3.

24 Manual del oficial subalterno, núm. 5, 1901,156 pp., en Colección Digital de la Universidad Autónoma de Nuevo León [consultado el 21/07/2014].

25 "Los manuales para los oficiales reservistas", Revista México Militar, Vol. 1, Núm. 19, $1^{\circ}$ de marzo de 1901, pp. 396-397. 
aspirantes. ${ }^{26}$ Aun así, los tomos no eran accesibles para cualquiera. Si consideramos que un periódico de la época, como El Imparcial, costaba un centavo, ${ }^{27}$ el manual completo (los dos tomos), cuyo precio sería de un peso, costaba 100 veces lo que un ejemplar del periódico. Un estudiante o profesionista podría adquirirlo, pero para un obrero o un campesino era seguramente muy caro.

De manera paralela a la publicación de manuales como éstos, los jóvenes interesados en pertenecer a la Segunda Reserva en la capital del país comenzaron a ser adiestrados por la propia Secretaría de Guerra (antes incluso de junio de 1901, mes en el que, de acuerdo con la Ley Orgánica, se abrirían formalmente los centros de instrucción). Una nota periodística informó que el primer contingente de aspirantes que estaba preparándose, en espera de que se pusiera en vigor la Ley Orgánica, sobrepasaba ya los doscientos jóvenes:

Entre las personas que reciben instrucción se cuentan gran número de profesionales e industriales que han concluido ya el aprendizaje de la primera parte de la escuela del soldado. / El número de los que actualmente reciben instrucción pasa de doscientos, y pronto, según se nos informa, se constituirá un grupo de caballería y otro de infantería, con diez aspirantes cada uno, para dar principio a la práctica de la orden de batalla. ${ }^{28}$

Estas cifras parecen exageradas, pues el número de aspirantes que finalmente fueron aceptados en la Segunda Reserva en el Distrito Federal, al menos durante el primer año, sería menor. De acuerdo con el cuadro 1, que se presenta a continuación, sólo ingresaron al cuerpo reservista 141 oficiales en el primer año de vida del proyecto. Es posible que El Imparcial abultara un poco los números para promover el proyecto. ¿O sería que el proceso de selección para ingresar a la Segunda Reserva era en verdad muy riguroso y que, aunque acudieron muchos a los entrenamientos previos, pocos lograron su ingreso? ${ }^{29}$

26 Idem.

27 Sobre el costo del ejemplar de El Imparcial, véase http://www.inehrm.gob.mx/Portal/ PtMain.php?pagina $=$ diasdemexico-detalle\&cat $=2$ [consultado el 21/07/2014].

28 "Los oficiales reservistas. Las juntas de los estados", El Imparcial, 7 de mayo de 1901, p. 3. 29 La prensa de la época da detalles de lo que fue el primer examen de selección para 


\section{La respuesta a la convocatoria: la oficialidad reservista en el Distrito Federal}

La respuesta a la convocatoria de la Secretaría de Guerra para alistarse en la Segunda Reserva parece haber sido importante en distintas regiones del país. En el Distrito Federal lo fue ciertamente; aun si las cifras de oficiales dados de alta no corresponden con los números que publicaba la prensa, existen registros oficiales de esa buena respuesta. La Secretaría de Guerra y Marina publicó el nombre y número de aspirantes aceptados en la Segunda Reserva desde julio de 1901 hasta el 31 de diciembre de 1902; sin embargo, en algunos de estos registros, el número de reservistas de las diversas entidades federativas se encuentra mezclado. Sólo es posible identificar el número y nombres de los reservistas que ingresaron al cuerpo en el Distrito Federal durante un año: de julio 1901 a junio $1902 .{ }^{30}$ De cualquier manera, y aunque no se tengan los datos para el último semestre de 1902, estos registros permiten valorar la importancia de la respuesta capitalina a la convocatoria para crear cuerpos reservistas. A continuación se presenta un cuadro con el número de aspirantes que fueron aceptados para ingresar a la Segunda Reserva en el Distrito Federal por cuerpo de adscripción, entre julio 1901 y junio 1902. (Ver cuadro 1).

\footnotetext{
ingresar a la Segunda Reserva. Por ejemplo, El Imparcial refiere que los aspirantes llegaban en "multitudes" a la prueba y que el examen consistía en un conjunto de preguntas orales sobre las ordenanzas y sobre cuestiones de estrategia militar, como los expuestos en el Manual del oficial subalterno. También afirma que había un jurado encargado de la evaluación y que el veredicto final acerca de la aceptación o no de un aspirante tendría que ser adoptado por unanimidad. La referencia a "multitudes" de aspirantes parece, nuevamente, una exageración, pero como intento mostrar en este artículo, sí hubo una buena respuesta a la convocatoria para la Segunda Reserva en el Distrito Federal. "Examen de Reservistas", El Imparcial, 19 de junio de 1901, p. 3.
}

30 Reyes, Memoria... 1901-1902, pp. 100-118. 
CuAdro 1. NÚMERo DE OFICIALES DADOS DE ALTA EN LA

SEgunda Reserva EN EL D. F. POR CUERPo DE ADSCRIPCIÓN

(JULIO 1901-JUNIO 1902)

\begin{tabular}{cc}
\hline Cuerpo de adscripción & Número de oficiales \\
\hline Caballería & 78 \\
\hline Infantería & 47 \\
\hline Servicios médicos & 3 \\
\hline Telegrafistas & 9 \\
\hline Farmacéuticos & 3 \\
\hline Ingenieros & 1 \\
\hline Total & $\mathbf{1 4 1}$ \\
\hline
\end{tabular}

Fuente: Reyes, Memoria... 1901-1902, t. I, pp. 65-118.

Los 141 oficiales dados de alta en el Distrito Federal durante el primer año de vida del proyecto fueron asignados, en su mayoría, al cuerpo de caballería (55\%); en segundo lugar, al cuerpo de infantería (33.5\%); el resto se repartió entre telegrafistas, servicios médicos, servicios farmacéuticos e ingenieros. Los cuerpos de caballería e infantería integraron una cantidad de oficiales por encima de los cuerpos especiales. Esto resulta normal si tenemos presente que el objetivo del proyecto reservista fue, desde un inicio, el fortalecimiento de los cuerpos tácticos del Ejército.

Al final de este artículo se incluye una tabla con los nombres de estos 141 subtenientes dados de alta en el Distrito Federal (Anexo 1). Entre ellos podemos identificar, a primera vista, a algunos personajes que posteriormente destacaron en diversos ámbitos de la vida pública nacional, por ejemplo, José Castellot Batalla, quien además de empresario, llegó a ser legislador federal y gobernador de Campeche en 1902. ${ }^{31}$ También figuran nombres como el de Venustiano Carranza, quien en el momento de ingresar a la Segunda Reserva rondaba los 32 años de edad. Carranza era hacendado y había fungido como alcalde de Cuatro Ciénegas, Coahuila (1887 y 1894-

31 Musacchio, Milenios, p. 517. 
1913); también ocupó una senaduría y llegó a ser gobernador interino de Coahuila durante el porfiriato. ${ }^{32}$

Joaquín Clausell es otro de los nombres que llaman la atención en la lista de reservistas del Distrito Federal. Este personaje, considerado el principal exponente de la pintura impresionista en México, había llegado a la capital de la república en la década de 1880, tras enfrentarse políticamente al gobernador de Campeche de ese entonces, Joaquín Baranda. Cursó la carrera de derecho y, en su juventud, fue caricaturista, colaborador de El Hijo del Abuizote — periódico censor del régimen-, así como de El Monitor Republicano y El Universal. Sus críticas al gobierno como periodista le habían costado el encarcelamiento varias veces, hasta que terminó exiliándose en Estados Unidos y en París, en 1896. Regresó a México en 1900, y aunque se dice que a partir de entonces sólo se dedicó a la pintura, su nombre se encuentra en los registros de la Segunda Reserva, publicados en las Memorias de la Secretaría de Guerra y Marina. ${ }^{33}$

Enrique Flores Magón es uno de los personajes que más llaman la atención en la referida lista, pues es bien conocida la postura antiporfirista que caracterizó a varios miembros de su familia. De hecho, Enrique mismo fue uno de los fundadores del periódico Regeneración, combativo órgano de prensa que atacó a Díaz directamente y que dedicó páginas a denunciar el proyecto reservista de Reyes. Enrique Flores Magón contaría con 25 años de edad aproximadamente cuando el proyecto reservista cobraba vida: ¿era un joven indeciso, contagiado por la "euforia" de la Segunda Reserva? ¿O sería más bien un espía de Regeneración, como llegó a acusársele en su momento? Difícil saberlo, pero su antiporfirismo acabó valiéndole el exilio en 1904.

Otro nombre destacado del listado de reservistas es el de Julio M. Limantour, hermano menor de José Yves Limantour —el secretario de

32 Años más tarde, durante el periodo revolucionario, Venustiano Carranza se uniría al antirreeleccionismo y ocuparía la Secretaría de Guerra y Marina como parte del gabinete provisional que formó Madero en Ciudad Juárez. En 1913 desconocería a Victoriano Huerta y encabezaría al Ejército Constitucionalista hasta el derrocamiento del usurpador. El 5 de febrero de 1917, como encargado provisional del poder ejecutivo del país, promulgaría una nueva Constitución, y el 6 del mismo mes convocaría a unas elecciones federales en las que resultaría electo presidente de la república.

33 Gantús y Gutiérrez, “Liberalismo y antiporfirismo”, pp. 155-182. 
Hacienda y líder del grupo político de los "científicos", principal rival de Bernardo Reyes en la carrera por posiciones de poder a nivel nacional. Además de terrateniente, Julio era un empresario moderno, con inversiones en la industria y en la banca; fue vicepresidente de la Cervecería Moctezuma y del Banco Internacional e Hipotecario de México; así como consejero y accionista de empresas como la Societé Financiere pour I'Industrie au Mexique, la compañía Nacional Mexicana de Dinamita y Explosivos, la Fábrica de Papel de San Rafael, la planta textil San Ildefonso, la fábrica de cigarros El Buen Tono y el Banco Nacional de México. Asimismo, fue socio de la firma bancaria y bursátil Hugo Scherer. ${ }^{34}$ ¿Por qué un joven de tal posición económica y hermano de José Yves Limantour, enemigo de Reyes, se sumaría a la Segunda Reserva? Es posible que el secretario de Hacienda y su grupo no hubieran estado en contra del proyecto reservista en sus inicios y que el joven Julio haya compartido el entusiasmo de tantos otros jóvenes por formar parte de un cuerpo militar de voluntarios.

Por último, al lado de estudiantes, pintores, académicos, empresarios y futuros políticos, en la lista de reservistas encontramos también el nombre de un personaje como Rodolfo Reyes, hijo mayor de Bernardo Reyes, quien había estudiado la carrera de leyes en la Escuela Superior de Jurisprudencia. Rodolfo Reyes se involucraría pronto en una guerra de prensa —que sería una auténtica "guerra sucia" - entre "científicos" y reyistas. ${ }^{35}$

Que la Segunda Reserva haya atraído a personajes tan relevantes como los que hemos retomado en esta lista habla de que, en efecto, fue un proyecto con un impacto importante entre las clases medias e, incluso, altas del Distrito Federal, así como de gran interés para una generación en búsqueda de espacios en la vida pública del país.

Tras esta valoración, vale la pena preguntarse también qué tan importante pudo haber sido la respuesta en el Distrito Federal a la convocatoria para formar la Segunda Reserva con respecto a la que hubo en otras entidades

34 Diccionario Porrúa, p. 2000.

35 Sobre esta "guerra sucia", dice Reyna Hinojosa: “[...] si existía cierta amabilidad entre Reyes y Limantour, la cordialidad era inexistente entre los grupos que rodeaban a ambos personajes. El grupo científico — Limantour - fue el primero en embestir y rápidamente atacó a Bernardo Reyes haciendo uso de la prensa adicta. [...] Como respuesta a estos ataques, el grupo de amigos de Bernardo Reyes pronto utilizó el mismo conducto, apareciendo en La Protesta los dardos periodísticos.” Reyna Hinojosa, El general Bernardo Reyes, pp.174-175. Esta guerra periodística preparó la caída de Reyes de la Secretaría de Guerra. 
del país. Las Memorias de la Secretaría de Guerra y Marina registran, para ese primer año de vida del proyecto, la emisión de un total de 1878 patentes de oficiales reservistas para todo México. ${ }^{36} 140$ de ellas corresponden al D. F.: el $7.4 \%$ del total. Jalisco superó a la capital del país con 175 oficiales, es decir, el 9.3\% del total. (Ver cuadro 2) ¿Hubo mejor respuesta en Jalisco que en el D. F.?

CuAdro 2. ENTIDAdES FEDERATIVAS CON MAYOR NÚMERo DE OFICIALES aportados a la Segunda Reserva entre el $1^{\circ}$ DE Julio de 1901 y EL 30 DE JUNIO DE 1902

\begin{tabular}{|c|c|c|c|c|}
\hline Columna 1 & Columna 2 & Columna 3 & Columna 4 & Columna 5 \\
\hline $\begin{array}{l}\text { Entidad } \\
\text { federativa }\end{array}$ & $\begin{array}{c}\text { Número } \\
\text { total de } \\
\text { habitantes } \\
\text { en la entidad } \\
\text { federativa }\end{array}$ & $\begin{array}{l}\text { Número total } \\
\text { de oficiales } \\
\text { dados de } \\
\text { alta en la } \\
\text { entidad } \\
\text { federativa }\end{array}$ & $\begin{array}{c}\text { Porcentaje } \\
\text { de oficiales } \\
\text { registrados } \\
\text { en la } \\
\text { Segunda } \\
\text { Reserva } \\
\text { por entidad } \\
\text { federativa } \\
\text { en términos } \\
\text { absolutos }\end{array}$ & $\begin{array}{c}\text { Porcentajes } \\
\text { de oficiales } \\
\text { reservistas } \\
\text { alistados en } \\
\text { la entidad } \\
\text { federativa en } \\
\text { relación con } \\
\text { el número de } \\
\text { habitantes de } \\
\text { la entidad }\end{array}$ \\
\hline $\begin{array}{l}\text { Distrito } \\
\text { Federal }\end{array}$ & 541516 & 140 & 7.4 & 0.025 \\
\hline Jalisco & 1153891 & 175 & 9.3 & 0.015 \\
\hline Nuevo León & 327937 & 73 & 3.8 & 0.022 \\
\hline Puebla & 1021133 & 87 & 4.6 & 0.008 \\
\hline $\begin{array}{l}\text { San Luis } \\
\text { Potosí }\end{array}$ & 575432 & 107 & 5.6 & 0.018 \\
\hline Veracruz & 981030 & 81 & 4.6 & 0.008 \\
\hline
\end{tabular}

Reyes, Memoria... 1901-1902, t. I, 364 p.; Estadísticas sociales, 249 p.

36 Reyes, Memoria... 1901-1902, t. I, 364 p. 
El cuadro 2 presenta las cifras de las entidades federativas en las que se dio de alta el mayor número de oficiales reservistas, entre el $1^{\circ}$ de julio de 1901 y el 30 de junio de 1902: Distrito Federal, Jalisco, Nuevo León, Puebla, San Luis Potosí y Veracruz. ${ }^{37}$ En este mismo cuadro hemos registrado el número total de habitantes de cada entidad seleccionada, el número de oficiales reservistas dados de alta en cada una de ellas entre julio de 1901 y junio de 1902, el porcentaje que representaron los oficiales alistados en cada una de esas entidades con respecto al total de los 1878 oficiales de la Segunda Reserva para esas fechas y, finalmente, el porcentaje de oficiales reservistas alistados en cada entidad federativa en relación con el número de habitantes de la entidad. Este conjunto de cifras permite ver el lugar significativo que tuvo el Distrito Federal en el reclutamiento de oficiales para la Segunda Reserva.

Es verdad que $7.4 \%$ de los 1,878 oficiales reservistas reclutados en el Distrito Federal el primer año del proyecto se encuentran por abajo de $9.3 \%$ de Jalisco, entidad de donde era originario Bernardo Reyes. En cualquier caso, ese porcentaje pone a la capital del país en segundo lugar, por encima de todos los demás estados, incluido el de Nuevo León, del que el general Bernardo Reyes había sido gobernador por tantos años.

Sin embargo, para una correcta valoración del número de oficiales reservistas en el Distrito Federal con respecto a otras entidades del país, existe una variable importante que debe ser tomada en cuenta y que hemos incluido en el cuadro 2: el número de habitantes que tenía cada una de las entidades federativas consideradas. Tomando en cuenta esta variable, hemos calculado el porcentaje que representó el número de oficiales reservistas reclutados en cada entidad en relación con el número de habitantes de cada una de ellas. ${ }^{38}$ Este nuevo cálculo cambia de manera importante la valoración que puede hacerse del peso que tuvo la capital del país en la creación de la Segunda Reserva. Efectivamente, Jalisco, que fue el estado que alistó el mayor número de oficiales en términos absolutos (175 entre julio de 1901

37 Las entidades expuestas en el cuadro 2 fueron seleccionadas por ser las de mayor número de oficiales reservistas aportados de los 1878 dados de alta entre el $1^{\circ}$ de julio de 1901 y el 30 de junio de 1902. Hemos recuperado estos datos para poder hacer una comparación y valoración de los encontrados para el Distrito Federal.

38 Estadísticas sociales, 249 p. Tomamos el número de habitantes por entidad federativa para el año de 1900, porque estas estadísticas carecen de registros para 1901 y 1902. 
y junio de 1902), pierde presencia al considerar esa cifra de cara al total de su población (0.015\%); es superado por Nuevo León (0.022\%), pero, sobre todo, por el Distrito Federal (0.025\%). Con la consideración de la variable del número de habitantes, la capital del país pasa a ser la entidad que, en términos relativos, aportó el mayor número de oficiales al cuerpo reservista.

\section{Los reservistas en preparación y la visión de la prensa}

Los reservistas — tanto aspirantes como oficiales ya integrados al cuerpotenían la obligación de acudir cada domingo a entrenar y "pasar revista", como se conocía al acto de presentarse al cuerpo. Se les citaba los fines de semana porque el proyecto consideraba que la instrucción militar no debería afectar las actividades económicas de sus miembros en tanto civiles y, precisamente, el domingo era un día en que la mayoría de los ciudadanos descansaba de sus trabajos cotidianos.

La primera academia adaptada para recibir reservistas en el Distrito Federal estuvo lista desde los meses iniciales de 1901 y fue la escuela de tiro de San Lázaro. En dicho recinto se concentraron los primeros contingentes de aspirantes y de oficiales graduados. Pero no fue el único lugar. Conforme el proyecto tomaba fuerza y atraía a más solicitantes y más subtenientes causaban alta como reservistas, se tuvieron que abrir más espacios para los entrenamientos dominicales. Entre junio de 1901 y diciembre de 1902, en el Distrito Federal se adaptaron y abrieron un total de nueve academias para entrenamiento de aspirantes y oficiales de la Segunda Reserva: San Lázaro, La Piedad, Tacubaya, San Joaquín, Santa Fe, Santa Julia, Azcapotzalco, Cuautepec de Madero y México. ${ }^{39}$

En estos recintos, los reservistas recibían un entrenamiento similar al de los militares de las filas permanentes. Desde luego, los resultados en términos de destrezas adquiridas o, al menos, del tiempo que tomaría alcanzarlas, serían necesariamente distintos, ya que los reservistas sólo

39 En el país se dispusieron 264 academias para entrenar reservistas. Los nueve recintos del DF parecen pocos frente a esa cifra, pero para una comparación justa tendríamos que considerar el tamaño de los recintos y la exigencia de abrir muchos en territorios extensos y mal comunicados, que no era el caso del D.F. No hemos hecho este ejercicio aquí, pero es muy posible que tales factores puedan explicar el desbalance apuntado. Para el número de academias para entrenar reservistas, véase Reyes, Memoria... 1901-1902, p. 364. 
podían practicar los domingos. Se llevaban a cabo ejercicios de marcha y tiro al blanco a grandes distancias, de avance de efectivos en formación de columna y de caballería, y prácticas de asistencia a soldados enfermos o heridos en lugares donde no hubiera hospitales y estudio de manuales. ${ }^{40}$ Los reservistas se ejercitaban también en simulacros de guerra. Estos simulacros eran prácticas en las que las filas nacionales representaban, sin los riesgos de una batalla real, un hecho de guerra. Se llevaban a cabo para probar algún armamento nuevo o bien para ensayar disciplina y conocimientos aprendidos durante la instrucción regular. Estos ejercicios demandaban mucha preparación previa, pues si bien eran simulaciones que no ponían en mayor riesgo la vida del soldado o del reservista, a veces había accidentes, con heridos y lesionados. Tal era la intensidad con que se practicaban esos ensayos bélicos.

Los simulacros acaparaban mucho la atención del público: "gran espectáculo de armas", los llamaba la prensa. Con frecuencia, se les programaba para realizarse en fechas de importancia cívica, como la celebración del aniversario de la independencia de México. De esa manera se conjuntaban entrenamiento y representación festiva, ejercicio castrense y desfile. En realidad, al secretario le interesó siempre mostrar públicamente su fuerza, por lo que la combinación parecía perfecta.

El Imparcial celebraba tanto representaciones y desfiles como toda actividad relativa a la Segunda Reserva. Pero no toda la prensa aplaudía por igual los simulacros y, en general, los actos de propaganda en torno a la Segunda Reserva. Las opiniones sobre estos ejercicios bélicos no siempre fueron halagadoras. Periódicos como El Diario del Hogar y Regeneración, críticos del gobierno, hacían crónicas diferentes a las de El Imparcial cuando de la Segunda Reserva se trataba. Por ejemplo, el 4 de agosto de 1901, se tiene noticia de que se llevó a cabo un simulacro de reservistas en el Distrito Federal. Mientras El Imparcial fue dando seguimiento a los entrenamientos, ensayos generales y hasta al plan de acciones, El Diario del Hogar publicó una nota de censura que ponía en tela de juicio no sólo la efectividad de

40 Reyes, Memoria... 1900-1901, Anexos número 3, 4, 5, 6, 7, 8, 9, 10 y 11 correspondientes a las circulares número 525, 262, 266, 281, 292, 291, 289, 297, 269 respectivamente, pp. 59-80; Reyes, Memoria... 1901-1902, Anexos número 94, 95, 96, y 97 correspondientes a las circulares número 307, 252, 323, 319 respectivamente, pp. 241-250. 
un ejercicio como los simulacros de guerra, sino el proyecto mismo de la Segunda Reserva. La nota de El Diario del Hogar decía así:

México, según la expresión de los tácticos que dirigen el departamento guerrero nacional, debe prevenirse para el caso de una guerra extranjera. ¿Es pues inminente el peligro que nos amenaza y tenemos que resolverlo en batallas campales? / Nosotros contestamos que no; y dado el caso fortuito de batirnos en defensa de nuestra nacionalidad, cla guerra de cuerpos de ejército es la que más conviene a esa defensa? También contestaremos que no, ¿por qué? Porque el enemigo que nos amague en son de conquista, sería numerosísimo y nosotros no tendríamos el número suficiente de hombres que oponerle y sería una temeridad el sacrificio de vidas tratando de contenerlo en batallas campales. ¿Qué habría necesidad de hacer? [...] / Educar al pueblo en las prácticas democráticas, hacerlo concurrir a las academias en donde se impregne su ánimo del santo efecto a la libertad, sacarlo del estado de abyección en el que lo tiene sujeto una política de exclusivismo, sería el mejor camino. ${ }^{41}$

La nota descalificaba al proyecto reservista y reclamaba, para la mejor defensa del país, ejercicios democráticos, no bélicos.

El periódico antiporfirista Regeneración, fundado por los hermanos Flores Magón, tomó posición frente a la Segunda Reserva. Regeneración denunció el proyecto como una maniobra del general Reyes para ganar partidarios con miras a la sucesión presidencial. Publicó, por ejemplo, un llamado a las personas de "buena fe" para que no se dejaran engañar por un proyecto como el de la Segunda Reserva, un proyecto que los utilizaría en favor de la dictadura. Bernardo Reyes, en su opinión, prolongaría la "tiranía" de Díaz por medios aún más "violentos". Los redactores del periódico estaban convencidos de que los jóvenes reservistas serían ocupados como escalón político del "ambicioso" Reyes para llegar a la presidencia de la república. ${ }^{42}$ El Diario del Hogar y Regeneración eran voces críticas frente a la Segunda Reserva, claramente opuestas a las de una prensa oficialista como El

41 "Sumario. ¿Nos prevenimos para la Guerra? Los simulacros ¿cuál conflicto guerrero nos amenaza? ¿Triunfaremos? Sí”, El Diario del Hogar, 30 de julio de 1901, p. 3.

42 "A los jóvenes reservistas", Regeneración, 15 de agosto de 1901, pp. 12-14. 
Imparcial. El Diario del Hogar se burlaba de los aspirantes y oficiales recién alistados; Regeneración invitaba abiertamente a los jóvenes a abandonar la institución reservista. ${ }^{43}$ Sin embargo, ni uno ni otro pusieron nunca en duda la exitosa respuesta que estaba encontrando la convocatoria para formar la Segunda Reserva.

La prensa crítica del régimen parece haberle hecho poca mella al proyecto, pero, de todas formas, la Segunda Reserva tuvo corta vida. El recelo de los grupos porfiristas rivales de Reyes llevaría a su cancelación.

\section{Consideraciones finales}

La llegada de Bernardo Reyes a la Secretaría de Guerra en 1900 fue un acontecimiento de mucho significado para la política del porfiriato tardío. Su presencia en el gabinete del presidente Díaz lo catapultó a la arena política nacional. Como secretario de Guerra comenzaría una reorganización del Ejército. Su papel de reformador le dio a Reyes popularidad entre los propios militares y, a la par, en el terreno de la política nacional. En efecto, lo mostró como un político hábil. En especial la Ley Orgánica del Ejército Nacional, promulgada en 1900, significó un paso importante en el proceso de centralización de las armas nacionales, pues logró poner prácticamente a todos los cuerpos armados bajo el mando directo de la Secretaría de Guerra y Marina.

Particularmente ambicioso fue el proyecto de creación de un cuerpo reservista con una nueva oficialidad compuesta por ciudadanos voluntarios. La Segunda Reserva parece haber sido bien recibida en el país — lo fue ciertamente en la capital - y dio a Reyes una popularidad insospechada entre algunos sectores de la sociedad. Un político respaldado por una fuerza como la que comenzaba a formarse con la Segunda Reserva preocupó mucho a sus rivales políticos, lo que terminaría costándole su puesto en el gabinete de Díaz, a sólo dos años de haber sido designado secretario de Estado.

$43 \mathrm{Al}$ margen de los beneficios reales que pudiera tener para México el contar con un cuerpo de reservistas en caso de una guerra extranjera, a los redactores de Regeneración les parecía más seria la amenaza de la llegada de otro militar al poder: el general Reyes. Y temían que pudiera hacerlo gracias a la fuerza adquirida a través de sus reservistas. "A los jóvenes reservistas", Regeneración, 15 de agosto de 1901, pp. 12-14. 
La respuesta a la convocatoria para la creación de la Segunda Reserva en el Distrito Federal fue ciertamente entusiasta. La publicación de listados de aspirantes y las noticias sobre los entrenamientos, simulacros y desfiles dan fe de ello. Estos testimonios, sumados a los listados de oficiales dados de alta que ofrecen las Memorias de la Secretaría de Guerra y Marina de 1900 a 1902, evidencian el buen arranque del proyecto en la capital del país. De hecho, el Distrito Federal parece haber sido magnífica arena para impulsar el proyecto reservista. En esta entidad se enlistaron 140 oficiales en el primer año de vida de la Segunda Reserva, el segundo contingente más importante del país. Y si consideramos el número de habitantes del Distrito Federal y hacemos una comparación con los enlistados en entidades con mayor población, la capital del país pasa a ocupar el primer lugar en respuesta a la convocatoria reservista. Igualmente, la presencia de personajes reconocidos en la vida política de la época como promotores de la Segunda Reserva, y los nombres en las listas de jóvenes oficiales reservistas que figurarían en la política nacional años más tarde, muestran la relevancia del proyecto y el impacto que comenzaba a tener.

Todo parece indicar que, al menos en la experiencia del Distrito Federal, el proyecto reservista fue bien acogido entre jóvenes inquietos de clase media y alta, atentos a oportunidades de participar en la vida pública; aunque también parece haber despertado el interés entre otros sectores urbanos, como artesanos y obreros.

En 1902 se llevó a cabo un impresionante desfile militar por el aniversario de la independencia de México. En dicho evento, el general Bernardo Reyes hizo marchar un contingente grande de oficiales y aspirantes reservistas por las calles del centro de la ciudad de México. La historiografía que relata tal acontecimiento no llega a ponerse de acuerdo acerca del número exacto de reservistas que participaron en aquel desfile ${ }^{44}$ sin embargo, todos los autores coinciden en que, en una postura arrogante por parte del general Bernardo Reyes, se convocó a los reservistas para hacer alarde de la fuerza política y militar que había adquirido desde su llegada al gabinete

44 Benavides Hinojosa, por ejemplo, menciona la presencia de un total de 16000 reservistas en el desfile, pero Alicia Hernández y Josefina G. de Arellano reconocen sólo 6000 . Rodolfo Reyes, hijo del general, escribe que fueron 20 000. Benavides Hinojosa, Bernardo Reyes, p. 247; Hernández Chávez, La tradición republicana, p. 148; García de Arellano, Bernardo Reyes, p. 45; Reyes, De mi vida, p. 28. 
presidencial. Cualquiera que haya sido el tamaño exacto del contingente que marchó aquel 16 de septiembre de 1902 frente a Palacio Nacional, y sin entrar a discutir aquí las intenciones del general Reyes, la acción dio pie a innumerables rumores sobre sus ambiciones de poder, así como a una auténtica campaña de grupos políticos rivales en su contra. En especial, los "científicos" comenzarían, cerca de Díaz, una guerra de prensa y de presiones que tuvo como eje el "peligro" de un hombre con tanto poder civil y militar como el secretario de Guerra.

Finalmente, el presidente Díaz decidió retirarle su apoyo al secretario de Guerra. El general Reyes renunció al cargo y regresó a la gubernatura de Nuevo León. Díaz quería mantenerlo leal al régimen y que no desapareciera del escenario público totalmente, porque era un buen contrapeso a las ambiciones de otros grupos, pero también buscó que perdiera su gran protagonismo en la escena política nacional.

De todas formas, Bernardo Reyes quedó terriblemente debilitado y su proyecto reservista acabaría con él. La Ley Orgánica de 1900, que había dado vida al proyecto de la Segunda Reserva, se mantuvo vigente, pero el cuerpo reservista fue pronto desmovilizado y el apartado referente a la organización de las reservas quedaría derogado en abril de $1904 .{ }^{45}$

La salida de Bernardo Reyes de la Secretaría de Guerra acusado, aunque no fuera formalmente, de utilizar a las reservas con fines de autopromoción política con miras a la sucesión presidencial, explica su renuncia. Sin embargo, y esto es lo que más me interesa, poco se ha tratado de profundizar en torno a la explicación del porqué el proyecto reservista tuvo tan buena respuesta a nivel nacional. ¿Es acaso que su éxito se debió a la popularidad previa del general Reyes? Más bien parece haber sido a la inversa. Si no, ¿por qué el gran interés por el proyecto en el Distrito Federal, donde Reyes no tenía el arraigo que poseía en el norte del país o en su natal Jalisco? Hay que tener bien presente que la Segunda Reserva prometió espacios a las generaciones jóvenes interesadas en la política, y creo que justo en esta premisa debe hallarse, aunque sea parcialmente, la explicación de la gran respuesta a la convocatoria en la capital del país.

45 "El General Díaz, el $1^{\circ}$ de abril de 1904, al abrir el $21^{\circ}$ Congreso Constitucional, el segundo periodo del segundo año de sus sesiones”, en Los presidentes de México, p. 694. 


\section{ANexo 1. Relación de oficiales Reservistas que fueron Dados DE ALTA ENTRE EL $1^{\circ}$ DE JULIO DE 1901 Y EL 30 DE JUNIO DE 1902 en el Distrito Federal}

\begin{tabular}{|c|c|c|}
\hline Alas, Juan Manuel & Martínez, Arnulfo & Jaramillo, Vicente \\
\hline Alonso, Conrado L. & Martínez, Conrado & Kegel, Alfonso J. \\
\hline $\begin{array}{l}\text { Álvarez del Castillo, } \\
\text { Gabriel }\end{array}$ & Martínez, Medardo & L- Prado, Ángel \\
\hline $\begin{array}{l}\text { Álvarez Rul y } \\
\text { Escalante, Carlos }\end{array}$ & $\begin{array}{l}\text { Mendoza, Francisco } \\
\text { de P. }\end{array}$ & Leguizamo, José E. de \\
\hline Antillón, Carlos & Moya Zorrilla, Víctor & López, Taide \\
\hline Arpide, Urbano & $\begin{array}{l}\text { Nájera y de Pindter, } \\
\text { Domingo J. }\end{array}$ & Martínez, Alberto \\
\hline Bribiesca, Jesús & Obscuras, José J. & Martínez, Luis E. \\
\hline Bulman, Manuel G. & Orellana, Adolfo & Méndez, Alfredo P. \\
\hline $\begin{array}{l}\text { Calderón y Paredes, } \\
\text { Rodolfo }\end{array}$ & Padilla, Conrado & Montañez, Vicente \\
\hline Cantoral, Mariano B. & Pérez Gálvez, Juan de & Ochoa, Eligio \\
\hline Carbajal, Luis R. & Pliego, Ismael & Orihuela, Enrique A. \\
\hline Carranza, Venustiano & Ponce, Fernando & Pedroza, Ricardo \\
\hline $\begin{array}{l}\text { Castañeda Ortiz, } \\
\text { Miguel }\end{array}$ & Ramos Arizpe, Rafael & Pérez Castro, Gonzalo \\
\hline Castañón, Fernando & $\begin{array}{l}\text { Ramos Pedrueza, } \\
\text { Antonio }\end{array}$ & Quiroz, Ignacio C. \\
\hline Castellot, José (h) & Renero, Manuel S. & Ramírez, Agustín \\
\hline Cervantes, Antonio & Reyes, Rodolfo & Ramírez, Rafael H. \\
\hline $\begin{array}{l}\text { Cervantes, Wenceslao } \\
\mathrm{H} \text {. }\end{array}$ & Rocha, Jesús M. & San Juan, Manuel H. \\
\hline Clausell, Joaquín & Rocha, Manuel & Sierra, Miguel \\
\hline Corona, Juan M. & $\begin{array}{l}\text { Robleda y Guerra, } \\
\text { Salvador }\end{array}$ & Solórzano, José \\
\hline Cruz, Emilio & Salazar, Manuel I. & Soriano, Manuel F. \\
\hline
\end{tabular}




\begin{tabular}{|c|c|c|}
\hline Cuevas, Niguel G. & Salazar, Miguel M. & Uribarri, Roberto \\
\hline Dahlhaus, Enrique & Tagle y Togno, Enrique & Vallarta, Luis C. \\
\hline Dahlhaus Osio, Emilio & Tavares. Nocilas & Vaquero, Manuel \\
\hline $\begin{array}{l}\text { Díaz Valadez, } \\
\text { Epigmenio }\end{array}$ & Villalón, Jerónimo C. & Velázquez, Manuel A \\
\hline Escamilla, Benjamín & Villamil, Antonio & Yáñez, Othón \\
\hline Escamilla, Eufemio & Villamil, Ricardo & Aguayo, Justo P. \\
\hline $\begin{array}{l}\text { Fernández Guerra, } \\
\text { Salvador }\end{array}$ & Záyago, Remigio & $\begin{array}{l}\text { Oñate, Raymundo } \\
\text { Miguel }\end{array}$ \\
\hline $\begin{array}{l}\text { Fernández, Salvador } \\
\text { Diego }\end{array}$ & Zertuche, Albino & Urritia, Pedro \\
\hline $\begin{array}{l}\text { Flores, Enrique } \\
\text { [Magón] }\end{array}$ & Adorno, Francisco & Echeveste, José M. \\
\hline Flores, Fernando & Alvarado, Julio & $\begin{array}{l}\text { Fuente Muñoz, } \\
\text { Manuel de la }\end{array}$ \\
\hline Flores, Francisco & Burgos, Ignacio & Gallegos, Manuel G. \\
\hline Galicia, José & Butrón, Eduardo M & Lara, Jesús B. \\
\hline $\begin{array}{l}\text { García Rebollo, } \\
\text { Lorenzo }\end{array}$ & Cárdenas, Gonzalo & López Guerrero, Carlos \\
\hline Gavaldón, Avelino & $\begin{array}{l}\text { Carreño, Alberto M. } \\
\text { [María] }\end{array}$ & Muñoz, Edmundo \\
\hline $\begin{array}{l}\text { Gómez Farías, } \\
\text { Salvador }\end{array}$ & $\begin{array}{l}\text { Castañeda y Guiard, } \\
\text { Arturo }\end{array}$ & Pérez Valiente, Luis \\
\hline González, Efigenio & Castaño, Natalio & $\begin{array}{l}\text { Sánchez Cordero, } \\
\text { Mariano }\end{array}$ \\
\hline González, Griseldo & Castro, Antonio G. & Téllez Durán, Ismael \\
\hline González, Gabriel E. & Castro, Miguel S. & $\begin{array}{l}\text { Mercenario, Esteban } \\
\text { A. }\end{array}$ \\
\hline González, Salvador & Chávez, Fernando & Montaño, Emilio F. \\
\hline Graef, Carlos & Elzaurdia, Fernando & Ramos, José \\
\hline $\begin{array}{l}\text { Gracia y Medrano, } \\
\text { Bernardo }\end{array}$ & Enríquez, Rodimiro & Villareal, Julián \\
\hline
\end{tabular}




\begin{tabular}{ll}
\hline Gutiérrez, Alfonso & Espinosa, Abelino \\
\hline Hernández, Enrique & Fernández, Manuel G. \\
\hline Islas y Bravo, Lino & $\begin{array}{l}\text { García Brito, José } \\
\text { Ricardo }\end{array}$ \\
\hline Larrañaga, Manuel & García Ravelo, Miguel \\
\hline Limantour, Julio M. & González, Fernando \\
\hline Lozano Saldaña, Rafael & González, Rafael \\
\hline Maldonado, Aurelio & Granados, José \\
\hline Mariscal y Piña, Alonso & Gutiérrez, José \\
\hline Márquez, José G. & Hernández, Jerónimo \\
\hline
\end{tabular}

Fuente: Reyes, Memoria... 1901-1902, t. I, pp. 65-118.

\section{Fuentes}

\section{Archivos}

AHD-SEDENA Archivo Histórico Digital de la Sedena

AHUNAM Archivo Histórico de la UnAm

\section{Hemerografía}

Boletín del Oficial Reservista. Ciudad de México (Consultado en el AGEv. Archivo General del Estado de Veracruz)

El Diario Oficial de la Federación. Ciudad de México.

El Imparcial. Ciudad de México.

El Diario del Hogar. Ciudad de México.

Regeneración. Ciudad de México (Consultado en el Archivo Electrónico

Ricardo Flores Magón, en línea: http://www.archivomagon.net)

Revista México Militar. Ciudad de México (Consultado en la Biblioteca

Sebastián Lerdo de Tejada) 


\section{Bibliografía}

Arellano, Josefina G. de, Bernardo Reyes y el movimiento reyista en México, México, INAH - Programa de Historia Indígena, 1982, 186 p. (Científica, 131)

Benavides, Rafael, La Prusia militar o sea de la organización completa de los Ejércitos de la confederación del norte, en tiempo de paz y guerra, deberes generales de los diversos grados superiores e inferiores, alistamiento, movilización, escuelas, grandes maniobras y descripción ilustrada de su material de guerra, según los datos más auténticos y recientes por el general mexicano Rafael Benavides, Nueva York, Hallet \& Breen, 1873, 704 p.

Benavides Hinojosa, Artemio, Bernardo Reyes, un liberal porfirista, México, Tusquets, 2009, 380 p.

Celis, Pedro, "Las fuerzas militares auxiliares y de reserva en México; 18211914", tesis de licenciatura en historia, Facultad de Filosofía y Letras de la UNAM, 2012, 140 p.

Diccionario Porrúa. Historia, biografía y geografía de México, $6^{a}$ ed., México, Porrúa, 1995, 4 vols.

Dublán, Manuel, Colección Legislativa Completa de la República Mexicana con todas las disposiciones expedidas para la Federación, Distrito Federal y Territorios. Año de 1900, continuación de la legislación mexicana de Dublán y Lozano, Tomo 32, Única edición oficial, arreglada en virtud de autorización especial de la Secretaría de Justicia e Instrucción Pública, por el Sr. Lic. Agustín Verdugo, México, Talleres Tipográficos de Arturo y Alfredo G. Cubas, Arco S. Agustín 3, 1904, 921 p.

Estadísticas sociales del porfiriato 1877-1910, México, Secretaría de Economía Dirección General de Estadística/Talleres Gráficos de la Nación, 1956, $249 \mathrm{p}$.

González Oropeza, Manuel, Los diputados de la nación, México, Cámara de Diputados del Congreso de la Unión/Secretaría de Gobernación, 1994. [Accesible en internet: http://www.diputados.gob.mx/cedia/biblio/ virtual/dip/dipna.htm].

Hernández Chávez, Alicia, “Origen y ocaso del ejército porfiriano”, Historia Mexicana, vol. xxxix, núm. 1, julio-sept. 1989, pp. 257-296.

Hernández Chávez, Alicia, La tradición republicana y el buen gobierno, México, El Colegio de México/Fondo de Cultura Económica, 1993. 224 p. 
Knight, Alan, La Revolución Mexicana. Delporfiriato al nuevo régimen constitucional, traducción de Argelia Castillo Cano, México, Fondo de Cultura Económica, 2010, 2 vols.

Los presidentes de México ante la nación. Informes, Manifiestos, y Documentos de 1821-1966, Cámara de Diputados XLVI Legislatura, Tomo II, Informes y Respuestas desde el $1^{\circ}$ de abril de 1876 hasta el 4 de noviembre de 1911, México, Cámara de Diputados, 1966, 943 p.

Manual del oficial subalterno, México, Talleres de Ramón de S. N., Callejón de Santa Inés núm. 5, 1901, 156 pp. [Accesible en internet: Colección Digital de la Universidad Autónoma de Nuevo León]

Musacchio, Humberto, Milenios de México, Diccionario enciclopédico de México, México, Hoja Casa Editorial, 1999, 1008 p.

Niemeyer Jr., E. V., El General Bernardo Reyes, Monterrey, Gobierno del Estado de Nuevo León/Centro de Estudios Humanísticos de la Universidad de Nuevo León, 1966, 261 p.

Reyes, Bernardo. El ejército mexicano. Obras completas T. 2, comp. Adalberto Arturo Madero Quiroga, Monterrey, Honorable Congreso del Estado de Nuevo León - LXVIII Legislatura, 2000, 335 p.

Reyes, Bernardo, Memoria de la Secretaría de Estado y del despacho de Guerra y Marina presentada al Congreso de la Unión por el Secretario del ramo, general de división Bernardo Reyes, comprende del $1^{\circ}$ de enero de 1900 al 30 de julio de 1901, México, Tip. de la Oficina Impresora de Estampillas, 1901, 2 tomos.

Reyes, Bernardo, Memoria de la Secretaría de Estado y del despacho de Guerra y Marina presentada al Congreso de la Unión por el Secretario del ramo, general de división Bernardo Reyes, comprende del $1^{\circ}$ de julio de 1901 al 31 de diciembre de 1902, México, Tip. de la Oficina Impresora de Estampillas, 1903, 2 tomos.

Reyes, Bernardo, Ensayo sobre un nuevo sistema de reclutamiento para el Ejército y organizaciones de la Guardia Nacional, San Luis Potosí, Impr. de Cávalos, 1885, 145 p.

Reyes, Rodolfo, De mi vida. Memorias politicas. Vol. 1, (1899-1913), México, Biblioteca Nueva Madrid, 1929, 241 p.

Reyna Hinojosa, Ramiro, El general Bernardo Reyes ¡Presente!, México, Universidad Autónoma de Nuevo León, 2011, 511 p.

Sánchez Rojas, Luis Ignacio, "La Segunda Reserva del Ejército en Veracruz. 
1900-1902", Ulúa. Revista de Historia, Sociedad y Cultura, julio-diciembre de 2010, pp. 133-157.

Sánchez López Marco Enrique, "La Segunda Reserva del Ejército Nacional en el Distrito Federal, 1900-1902", tesis de licenciatura en historia, Facultad de Filosofía y Letras de la UNAM, 2014, 139p. 